\title{
Comparación e Inferencia en la Categorización de Artefactos No Familiares: Un Estudio con Niños Pequeños
}

\section{Comparison and Inference in the Categorization of Non- Familiar Artifacts: A Study With Young Children}

\author{
Andrea Sabina Taverna y Olga Alicia Peralta \\ Consejo Nacional de Investigaciones Científicas y Técnicas
}

\begin{abstract}
Investigaciones sobre el desarrollo de categorías muestran que procesos cognitivos como la comparación y la inferencia promueven el conocimiento conceptual. Este artículo reporta el estudio de estos procesos en la categorización de objetos no familiares, utilizando una prueba de elecciones forzosas y palabra extendida, manipulando propiedades funcionales de objetos nuevos. Utilizando una muestra accidental, el Estudio 1 examinó si 42 niños de 3 a 5 años extendían un nombre nuevo a un artefacto no familiar en base a su función, encontrándose un desempeño exitoso solo a los 5 años. El Estudio 2 investigó la comparación en la formación de categorías en 48 niños de 4 años y en el Estudio 3 se estudiaron los efectos de la inferencia en 24 niños de 4 años. Únicamente los niños que compararon artefactos bajo un rótulo común sortearon el sesgo perceptivo, formando categorías nuevas, no así los que fueron invitados a realizar inferencias en base a propiedades. Se discuten los alcances de la comparación y las limitaciones de la inferencia en la categorización de objetos en ausencia de conocimiento conceptual previo.
\end{abstract}

Palabras clave: comparación, inferencia, categorías no familiares, artefactos

\begin{abstract}
Prior research on category development has shown that cognitive processes such as comparison and inductive inference promote conceptual development, highlighting particular aspects of children's existing conceptual knowledge. This paper reports the study of children's comparison and inductive inference in the categorization of non-familiar objects. It used a forced-choice and word extension task with novel artifacts, manipulating their functional properties. Using an accidental sample, Study 1 assessed whether 2 to 5 years old children extended a novel word to a non-familiar artifact based of its function. It was found that only 5-year-olds succeeded. Study 2 tested the effects of comparison in the formation of categories in 48 4-year-olds and Study 3 the effects of inference in 24 4-year-old children. Only children who compared artifact-members under a common label went beyond the perceptual bias and formed new categories, but not the children who were invited to make property-based inference. Results are discussed concerning the extent of comparison and the restrictions of property-based inference in the categorization of objects with no pre-existing conceptual knowledge.
\end{abstract}

Keywords: comparison, conceptual-based inference, non-familiar categories, artifacts

¿Cómo aprenden los niños la estructura conceptual? Numerosas investigaciones han demostrado que incluso los bebés comprenden la naturaleza de conceptos y categorías (Mandler, 2004; Mareschal \& Quinn, 2001), ocurriendo luego progresos sustanciales durante la niñez (Mandler, 1992; Vygotsky, 1962).

Andrea Sabina Taverna y Olga Alicia Peralta, Instituto Rosario de Investigaciones en Ciencias de la Educación -IRICE (Consejo Nacional de Investigaciones Científicas y Técnicas - CONICET), Rosario, Argentina.

Esta investigación fue financiada por una Beca Doctoral del CONICET, otorgada a la primera autora bajo la dirección de la segunda, y por subsidios de la Agencia Nacional de Promoción Científica y Tecnológica (ANPCYT) y del CONICET, otorgados a la segunda autora.

Las autoras agradecen a los niños e instituciones participantes.

La correspondencia relativa a este artículo debe ser dirigida a Olga Alicia Peralta, Instituto Rosario de Investigaciones en Ciencias de la Educación, 27 de Febrero 210 bis. 2000, Rosario, Argentina. E-mail: peralta@irice-conicet.gov.ar 
Muchos estudios han utilizado la tarea de palabra extendida como herramienta para explorar la comprensión de conceptos. En ella se nombra un ejemplar de una clase con una palabra nueva y se pide al niño que extienda esa etiqueta a otros ejemplares posibles. Estas investigaciones demostraron que los preescolares pueden agrupar objetos según una gran variedad de criterios, por ejemplo, relaciones temáticas, pero, cuando se introduce una etiqueta nueva, tienden a aplicarla a cosas del mismo tipo (Imai, Gentner \& Uchida, 1994; Markman \& Hutchinson, 1984). Este fenómeno ha suscitado gran interés ya que parece ofrecer una ventana hacia un mecanismo por el cual los niños pueden adquirir el dominio de las categorías que caracterizan su mundo.

Utilizando este paradigma, numerosos estudios han mostrado que los niños basan sus categorizaciones primeramente en aspectos perceptivos como la forma (redonda) y características salientes de los objetos (ruedas) antes que en el conocimiento conceptual (Baldwin, 1992; Gentner, 1978; Graham \& Diesendruck, 2010; Graham, Kilbreath \& Welder, 2004). Pero también se ha demostrado que este sesgo perceptivo no es inviolable y que los niños pueden reconocer la estructura conceptual profunda si se los alienta a utilizar mecanismos que se hallan al servicio del aprendizaje de categorías (Booth, Waxman \& Huang, 2005; Namy \& Gentner, 2002; Taverna \& Peralta, 2007, en prensa).

Investigaciones centradas en procesos de inferencia inductiva sugieren que los niños pequeños realizan proyecciones entre miembros de una categoría, no solo en base a información perceptiva sino guiados por expectativas taxonómicas. Por ejemplo, si se les dice que un ave (flamenco) posee determinada propiedad (tiene leucocitos dentro), son más propensos a inferir que un segundo animal (tordo) con la misma etiqueta posee también tal propiedad y no otro animal (murciélago) que no recibe esa etiqueta, incluso cuando la información perceptiva entre los miembros se halla en conflicto (Gelman \& Coley, 1990; Gelman \& Markman, 1986). Algunos estudios han explorado la inferencia en el proceso inverso: inferir nuevas categorías a partir de propiedades específicas (Booth et al., 2005; Gelman, Collman \& Maccoby, 1986). En ellos, cuando a una entidad nueva se le aplica una etiqueta nueva y se la describe utilizando información conceptual (tiene ojos, entonces puede ver), incluso niños muy pequeños extienden esa etiqueta sobre la base de la información conceptual.

Por otra parte, importantes investigaciones centradas en el aprendizaje por analogía (Gentner \& Namy, 1999; Graham, Namy, Gentner \& Meagher, 2010; Namy \& Gentner, 2002) han mostrado que la comparación de ejemplares-categoriales bajo un rótulo común permite ir más allá de sus propiedades superficiales y detectar relaciones conceptuales subyacentes. Por ejemplo, si a los niños se les presentan dos entidades categoriales (manzana, fresa) con una etiqueta común ("blicket") serán más propensos a extender la etiqueta a otro miembro de la categoría (uvas) y no a una entidad perceptivamente similar (pelota). En cambio, si se les presenta solo una entidad, son más proclives a extender la etiqueta en base a relaciones perceptivas. El acto de examinar dos o más entidades permite a los niños evaluar regularidades y diferencias que no son inmediatamente evidentes ante un solo ejemplar. Numerosas investigaciones han documentado los beneficios de la comparación en el aprendizaje en diferentes dominios, tanto en niños como en adultos (Loewenstein \& Gentner, 2001; Wang \& Baillargeon, 2008).

En suma, utilizando la inferencia o la comparación, los niños pueden detectar nuevas relaciones entre ejemplares categoriales, lo que posibilita el desarrollo del conocimiento conceptual.

Las investigaciones revisadas se focalizaron principalmente en conceptos y categorías conocidos para los niños (e.g., animal), por lo que la comparación y la inferencia actúan enfatizando aspectos de conceptos que los niños ya poseen. Sin embargo, dado que existen muchos objetos desconocidos o no familiares para los niños, ¿la comparación y la inferencia también podrían generar un conocimiento conceptual nuevo, además de enfatizar el pre-existente?

En este sentido, Hammer, Diesendruck, Weinshall y Hochstein (2009) ilustraron la utilización de la comparación en la extracción de una relación entre objetos desconocidos por parte 
de adultos y niños de seis años. También Graham et al. (2010) mostraron los beneficios de la comparación en la categorización de objetos desconocidos que compartían la textura (rugoso) en niños de cuatro años. Los niños que compararon dos objetos extendieron la etiqueta en base a la textura y no a la forma, mientras que los que habían visto uno solo categorizaron en base a la forma. Si bien este trabajo muestra el rol de la comparación en la categorización de objetos nuevos en edad preescolar, el mismo ilustra relaciones perceptivas, como la textura.

Con respecto al papel de la inferencia, Farrar, Raney y Boyer (1992) mostraron un bajo rendimiento en niños preescolares y escolares en la habilidad para inferir conceptos nuevos que representaban animales utilizando información conceptual ("tiene leucocitos en el interior"). Sin embargo, Truxaw, Krasnow, Woods y German (2006) reportaron que niños de cuatro años fueron exitosos infiriendo categorías de artefactos no familiares en base a la información funcional en respuesta a diferentes tipos de instrucción. En tanto ambos estudios arrojan resultados contradictorios, la habilidad de los niños para inferir categorías desconocidas en base a propiedades permanece poco clara.

Precisamente, en la presente investigación investigamos el rol de la comparación y la inferencia en la extensión de nombres nuevos para objetos o artefactos desconocidos. Diseñamos los artefactos en base a los utilizados por Truxaw et al. (2006) y siguiendo una serie de criterios. En primer lugar, a los objetos los consideramos miembros de un tipo conceptual por poseer una función común con otros artefactos-miembros y por portar el mismo nombre. Para ello construimos los objetos de manera que parecieran artefactos diseñados intencionalmente para cumplir funciones simples y atractivas para los niños. A los artefactos con la misma función les asignamos un nombre común desconocido, a fin de evitar que portaran un significado a priori.

En segundo lugar, consideramos que el uso de la información funcional puede ser entendido como evidencia de categorías conceptuales cuando supera el conjunto de aspectos perceptivos o superficiales funcionalmente irrelevantes (Kemler Nelson, Russell, Duke \& Jones, 2000). Por lo tanto, los objetos debieron variar independientemente tanto en los aspectos perceptivos como funcionales, ya que, si ambos variaran conjuntamente, resultaría imposible determinar si la información funcional supera la información perceptiva y se constituye como base para utilizar los nombres de los artefactos.

Tercero, las categorías no debían responder al criterio de familiaridad. Si bien los niños pueden poseer conocimiento acerca de los principios físicos de los artefactos, a partir de los cuales se asocian sus funciones (e.g., conocer que la propiedad de un espejo es la de reflejar), ello no implica reconocer a los artefactos que las presentan como miembros de una categoría en tanto tipo conceptual.

Un aspecto importante fue determinar la edad en la que los niños podrían necesitar de la comparación o la inferencia para interpretar el significado de una palabra referida a un artefacto desconocido. Los estudios evolutivos clásicos típicamente presentan al niño un artefacto desconocido al cual se le aplica un nombre nuevo, pidiéndosele que extienda ese nombre a otros candidatos posibles: (a) perceptivamente similares al artefacto modelo, pero que cumplen una función diferente o (b) perceptivamente diferentes, pero que cumplen la misma función. Este tipo de investigaciones ha arribado a resultados contradictorios. Por ejemplo, tanto el clásico estudio de Gentner (1978) como estudios más recientes (Sloutsky, 2003) mostraron que los niños de dos a cinco años extienden el nombre en base a aspectos perceptivos del artefacto, mientras que los mayores de cinco años y los adultos lo hacen en base a su función. Por otro lado, otras investigaciones revelaron que bajo ciertas circunstancias (manipulación de la información funcional, instrucción) los niños de dos a cuatro años evidencian sensibilidad a la función de los objetos (Diesendruck, Markson \& Bloom, 2003; Kemler Nelson, Herron \& Morris, 2002).

En consecuencia, como primer objetivo consideramos necesario determinar diferencias evolutivas en la habilidad para extender el significado de una palabra en base a la información funcional, a fin de definir a qué edad sería apropiado estudiar el rol de la comparación e infe- 
rencia en la formación de categorías no familiares. En el Estudio 1 examinamos a qué edad los niños detectan por sí solos información funcional al extender el significado de una palabra. En el Estudio 2 investigamos los efectos de la comparación en la formación de categorías de artefactos y en el Estudio 3 nos centramos en el proceso de inferencia en base a propiedades.

\section{Estudio 1}

\section{Método}

Participantes. Trabajamos con una muestra accidental de 42 niños de tres grupos de edad: 15 de tres años (36-39 meses, $M=36,2, D E=2,1)$, 12 de cuatro años (47-49 meses, $M=$ $48,3, D E=0,7)$ y 15 de cinco años (59-62 meses, $M=60,3, D E=2,2)$. En este estudio y en los siguientes contactamos a los niños a través de sus instituciones educativas. Contactamos cuatro instituciones en base a que a ellas acuden niños de sectores medios urbanos de la ciudad; tres accedieron a participar.

Materiales. Construimos 18 objetos tridimensionales de cartulina desconocidos para los niños (artefactos). Organizamos los objetos en seis subpruebas con tres objetos cada una. Cada subprueba representaba una categoría que definimos por la función : (a) Huc: objetos cuya función es imantar, con una superficie de metal interna no visible en uno de sus lados para atraer objetos de metal; (b) Laky: objetos para verter, con un vertedor en el ángulo superior derecho; (c) Dax: objetos para guardar, con una ranura para introducir objetos pequeños; (d) Tuni: objetos cuya función es hacer sonido, con pequeñas unidades en su interior que suenan al agitarlas; (e) Nec: objetos para reflejar, con un pequeño espejo en uno de los lados y (f) Pong: objetos que sirven para abrir y cerrar. Una lista de los ítems de cada subprueba puede observarse en la Tabla 1.

Tabla 1

Lista de Ítems Utilizados en el Estudio 1, Estudio 2 en las Condiciones Sin Comparación (con y sin Palabra) y en el Estudio 3

\begin{tabular}{lll}
\hline \multirow{2}{*}{ Artefacto base } & \multicolumn{2}{c}{ Alternativas } \\
\cline { 2 - 3 } & \multicolumn{1}{c}{ Perceptiva } & \multicolumn{1}{c}{ Funcional } \\
\hline Huc - rectangular & rectangular & piramidal \\
colgar & imantar \\
Laky - cuadrado & cuadrado & cilindro \\
verter & hacer ruido & verter \\
Dax - piramidal & piramidal & rectangular \\
guardar & abrir y cerrar & guardar \\
Tuni - hexagonal & hexagonal & cuadrado \\
hacer ruido & imantar & hacer ruido \\
Nec - pentagonal & pentagonal & cilindro \\
reflejar & guardar & reflejar \\
Pong - rectangular & rectangular & pirámide \\
abrir y cerrar & reflejar & abrir y cerrar \\
\hline
\end{tabular}

Nota. La letra normal indica la forma de los artefactos y la cursiva, la función que cumplen. 
Utilizamos una versión del paradigma de extensión de la palabra (Gentner, 1978). Mostramos al niño tres artefactos: el modelo (e.g., artefacto rectangular cuya función es guardar) y dos alternativas. La alternativa funcional compartía la misma función con el artefacto modelo, pero era perceptivamente diferente (artefacto con forma piramidal que sirve para guardar). La alternativa perceptiva compartía similitudes perceptivas (forma) con el artefacto modelo, pero no cumplía la misma función (artefacto rectangular cuya función es reflejar). La Figura 1 muestra un ejemplo de los estímulos.

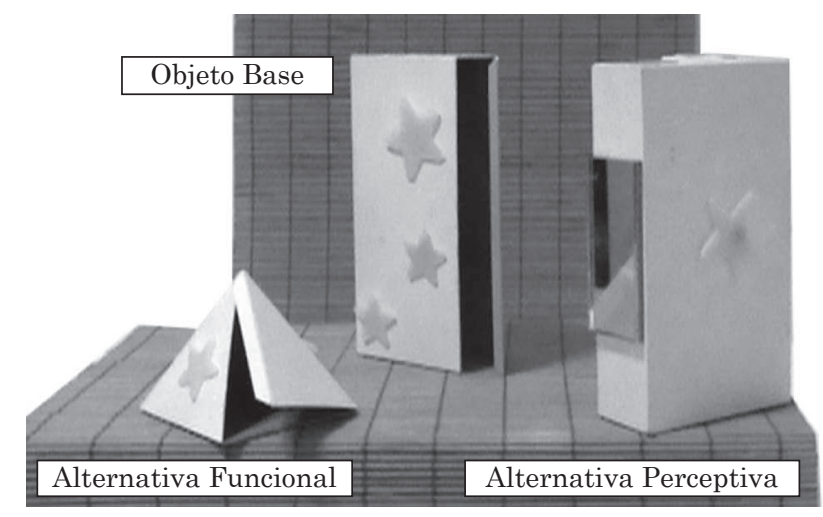

Figura 1. Materiales utilizados en el Estudio 1.

Procedimiento. Los niños de todos los estudios contaron con el consentimiento informado, escrito y firmado de sus padres.

Los niños fueron evaluados individualmente en una sala de la escuela. En este estudio y en los siguientes utilizamos un juguete, el marciano Marvin, para ayudar y alentar a los niños en la tarea, y una esfera de $35 \mathrm{~cm}$ de diámetro, el planeta de Marvin, que contenía los artefactos. El estudio consistió en dos fases: ensayo y evaluación. El propósito del ensayo fue introducir los materiales y familiarizar a los niños con la tarea. Primero, la experimentadora presentó al niño al marciano Marvin y su planeta, diciéndole que iba a aprender nombres en el idioma de Marvin para distintas cosas de su planeta. Le mostró un autito de juguete diciendo: “¿Ves esto? Es un Tini en el idioma de Marvin”. Luego le pidió que repitiera la etiqueta nueva y, apoyando dos alternativas, una idéntica al objeto modelo (auto) y otra sin relación alguna con él (silla), dijo: “¿Me puedes decir cuál de estos dos es también un Tini?” Ningún niño falló en seleccionar la alternativa idéntica.

Siguió la evaluación. Primero, la experimentadora dijo al niño que iban a ver unos objetos especiales construidos por Marvin. Utilizamos el mismo procedimiento que en el ensayo, excepto que ahora las subpruebas contenían el artefacto modelo y las alternativas funcional y perceptiva, mostrándose sus respectivas funciones sin explicitarlas verbalmente. Por ejemplo, la experimentadora presentaba el primer artefacto base (Dax: función guardar/forma piramidal), diciendo: “¿Ves esto? Esto es un Dax, en el idioma de Marvin”, mientras mostraba la función, colocando un pequeño botón dentro de la ranura. Luego, pedía al niño que repitiera la etiqueta nueva. Inmediatamente apoyaba sobre la mesa la alternativa perceptiva, mostrando su función (abrir y cerrar) y la funcional, mostrando su función (guardar). En ningún caso la experimentadora aludía verbalmente a la función de los artefactos. Luego preguntaba: ¿Cuál de estos dos es también un Dax?" Los niños fueron alentados, pero no recibieron corrección o asistencia alguna.

El orden de presentación de las subpruebas fue contrabalanceado. La ubicación derechaizquierda de las alternativas con relación al niño fue asignada al azar en cada subprueba. 
Análisis de Datos. La variable dependiente fue la proporción de respuestas funcionales seleccionadas (primera elección). Primero analizamos el desempeño de los niños contra el azar $(0,50)$, utilizando la prueba Binomial. Luego comparamos la ejecución de los niños por edad, mediante $\chi^{2}$.

\section{Resultados}

La proporción de alternativas funcionales seleccionadas fue $29,9 \%$ a los tres años, 40,3\% a los cuatro años y 70,0\% a los cinco años (Figura 2). A los tres años el desempeño de los niños estuvo por debajo del nivel del azar ( $p<0,001)$, a los cuatro años, al nivel del azar $(p=0,100) \mathrm{y}$ a los cinco, por encima $(p<0,001)$.

Luego comparamos la ejecución de los niños por edad, encontrando diferencias significativas entre los grupos: $\chi^{2}(2, N=42)=31,7, p<0,001$. Análisis subsiguientes revelaron diferencias significativas entre el grupo de tres y el de cinco años, $\chi^{2}(1, N=30)=29,7, p<0,001$, y entre el de cuatro y el de cinco años, $\chi^{2}(1, N=27)=29,7, p<0,001$, pero no entre los grupos de tres y cuatro años, $\chi^{2}(1, N=27)=2,1, p=0,100$.

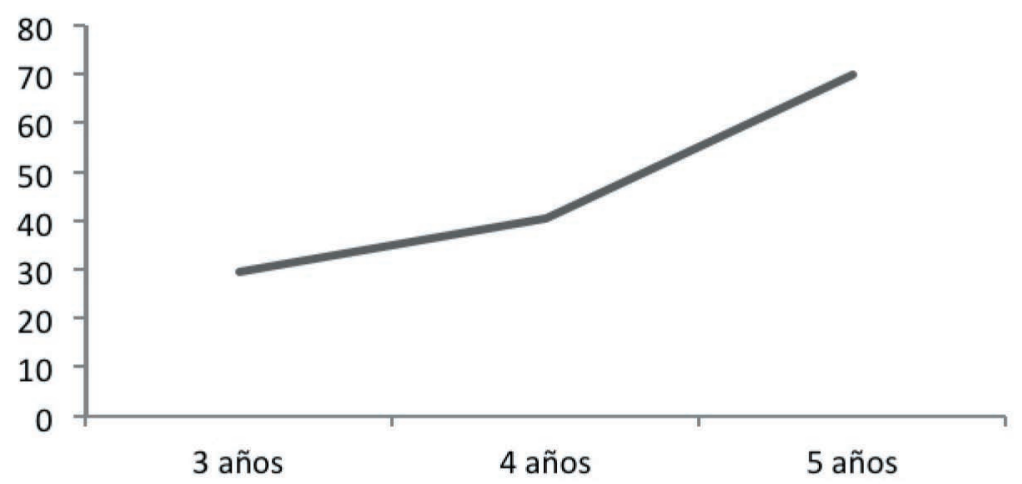

Figura 2. Proporción de respuestas funcionales por grupo de edad.

Estos resultados muestran que la mayoría de los niños de tres y cuatro años no reconoció relaciones funcionales entre los objetos; más aún, las respuestas de los niños de tres años fueron predominantemente perceptivas. En cambio, los niños de cinco años seleccionaron más alternativas funcionales, detectando la función de los artefactos al extender el significado del rótulo nuevo.

En un análisis complementario evaluamos si el rendimiento de los niños de cinco años fue resultado de su desempeño conceptual o si fue producto de un aprendizaje durante la tarea. Para ello comparamos la proporción de respuestas funcionales entre las subpruebas. La prueba Mac Nemar para muestra relacionadas no arrojó diferencias ni entre la primera $(46,6 \%)$ y la tercera subprueba $(73,3 \%), p=0,20$, ni entre la tercera $(73,3 \%)$ y la última $(66,6 \%), p>0,99$, lo cual indicaría una ausencia de aprendizaje por exposición a la tarea.

\section{Discusión}

Los niños de tres años se focalizaron predominantemente en las regularidades perceptivas al extender el significado de un nombre, mientras que los de cuatro no mostraron un patrón definido. Solo los niños de cinco años categorizaron los artefactos desconocidos en base a su función, esto es, utilizaron la información funcional, superando los aspectos perceptivos funcio- 
nalmente irrelevantes. Estos resultados son consistentes con los reportados por investigaciones que muestran que los niños pequeños tienden a utilizar aspectos salientes de la apariencia de los objetos como base para categorizar y que la información funcional llega a ser central más adelante (e.g., Gentner, 1978). Cabe destacar que la preeminencia del sesgo perceptivo en la categorización de objetos no implica que los niños pequeños sean insensibles a los aspectos funcionales, sino que cuando se les solicita que extiendan una palabra con un conocimiento incompleto o fragmentario acerca de las propiedades conceptuales del referente, la utilización del criterio perceptivo es la estrategia predominante. En esta línea, Imai et al. (1994) sugieren que una de las razones del énfasis en las respuestas perceptivas sería que a edades tempranas los niños están acostumbrados a adquirir términos de nivel básico en los cuales los aspectos perceptivos predicen de manera altamente confiable la estructura conceptual subyacente.

La evidencia encontrada se halla en consonancia con lo reportado por numerosos estudios con categorías familiares que muestran un incremento en el reconocimiento de relaciones conceptuales profundas y una correspondiente reducción de la detección de regularidades perceptivas con la edad (Gentner, Rattermann, Markman \& Kotovsky, 1995; Imai et al., 1994; Taverna \& Peralta, 2007, en prensa).

Teniendo en cuenta que las categorías que aquí estudiamos fueron categorías no familiares, tanto para los niños menores como para los mayores, los resultados amplían y apoyan la hipótesis del cambio relacional (Kotovsky \& Gentner, 1996). De acuerdo a esta hipótesis, para los niños pequeños las relaciones entre los objetos son altamente conservadoras y perceptivamente limitadas. Solo más tarde los niños pueden detectar sistemas de relaciones, independientemente de los atributos de los objetos, es decir, su sentido de tipo se hace más abstracto. Estas ideas, entonces, también pueden aplicarse a la formación de categorías de artefactos desconocidos, ya que su evolución parece seguir el mismo patrón. La hipótesis del cambio relacional es consistente con otros patrones observados en el desarrollo cognitivo, como las hipótesis del cambio perceptivo-funcional (Bruner, Goodnow \& Austin, 1956), el cambio de la caracterización-definición (Keil, 1996) y el perceptivo-taxonómico (Imai et al., 1994).

\section{Estudio 2}

El objetivo central de este estudio fue detectar si el mecanismo de comparación, que ha demostrado promover el conocimiento conceptual para objetos conocidos, también facilita la detección de la información funcional de artefactos no familiares.

Evaluamos el impacto de la comparación en niños de cuatro años, en virtud de que en el estudio anterior encontramos que recién a los cinco años los niños detectaron relaciones funcionales como base para sus categorizaciones.

Utilizamos una tarea de palabra extendida, similar a la empleada en el estudio anterior. Para ello manipulamos experimentalmente la posibilidad de que los niños comparasen artefactos del mismo tipo bajo un nombre común, presentándoles un solo objeto modelo (condición sin comparación) o dos (condición con comparación), antes de extender el significado de una palabra a una alternativa. Hipotetizamos que si la comparación de dos artefactos de un mismo tipo conceptual promueve la detección de relaciones profundas y abstractas (la función), al ver dos instancias de una misma categoría bajo una etiqueta común, los niños son más propensos a seleccionar una alternativa funcional que una perceptiva. En contraste, los niños que ven un solo artefacto modelo se focalizan predominantemente en relaciones perceptivas.

También indagamos si el efecto de la comparación en la detección de información funcional con artefactos no familiares ocurre solamente en un contexto lingüístico. Existe evidencia que muestra que la comparación facilita el aprendizaje de categorías familiares inclusive en contextos no lingüísticos (Gentner \& Namy, 1999; Kotovsky \& Gentner, 1996). Sin embargo, un estudio reciente con entidades no familiares que variaban en cuanto a propiedades perceptivas 
(textura y forma) también reveló que la efectividad de este mecanismo se ve acentuada por el efecto del nombre (Graham et al., 2010).

Teniendo en cuenta que en el presente estudio indagamos el rol de la comparación en la categorización de artefactos no familiares que varían en cuanto a propiedades profundas (función), hipotetizamos que el efecto de la comparación se ve apoyado por el input lingüístico. Para dilucidar si el efecto de la comparación en la promoción de un conocimiento conceptual nuevo es independiente o no del contexto lingüístico, presentamos a los niños los artefactos con (condiciones con etiqueta) o sin un nombre (condiciones sin etiqueta).

\section{Método}

Participantes. Participaron 48 niños de cuatro años divididos en cuatro grupos de 12 niños cada uno. Cada grupo fue testeado en una condición experimental: (a) Comparación con etiqueta (47-50 meses, $M=48,7, D E=1,1)$; (b) Comparación sin etiqueta (47-50 meses, $M=49$, $D E=1,6)$; (c) Sin comparación con etiqueta (47-49 meses, $M=48,3, D E=0,7)$ y (d) Sin comparación sin etiqueta (47-50 meses, $M=48,54, D E=1,7)$. Los datos de la condición c) provienen del grupo de niños de cuatro años del Estudio 1.

Materiales. También utilizamos al marciano Marvin y su planeta. Empleamos las seis subpruebas del estudio anterior; a cada subprueba le adicionamos un nuevo artefacto modelo diseñado con el mismo criterio, resultando un total de seis subpruebas con cuatro artefactos cada una (dos artefactos modelo y dos alternativas). Las Tablas 1 y 2 muestran las seis subpruebas con sus respectivos ítems por condición. La Figura 3 muestra un ejemplo de los estímulos utilizados.

Tabla 2

Lista de Ítems Utilizados en el Estudio 2 en las Condiciones Con Comparación (con y sin Palabra)

\begin{tabular}{llll}
\hline \multicolumn{2}{c}{ Subpruebas } & \multicolumn{2}{c}{ Alternativas } \\
\hline \multicolumn{1}{c}{ Artefacto base 1} & \multicolumn{1}{c}{ Artefacto base 2} & \multicolumn{1}{c}{ Perceptiva } & \multicolumn{1}{c}{ Funcional } \\
\hline Huc - rectangular & rectangular grande & rectangular & piramidal \\
imantar & imantar & colgar & imantar \\
Laky - cuadrado & cuadrado pequeño & cuadrado & cilindro \\
verter & verter & hacer ruido & verter \\
Dax - piramidal & piramidal doble & piramidal & rectangular \\
guardar & guardar & abrir y cerrar & guardar \\
Tuni - hexagonal & hexagonal pequeño & hexagonal & cuadrado \\
hacer ruido & hacer ruido & imantar & hacer ruido \\
Nec - pentagonal & pentagonal grande & pentagonal & cilindro \\
reflejar & reflejar & guardar & reflejar \\
Pong - rectangular & rectangular pequeño & rectangular & pirámide \\
abrir y cerrar & abrir y cerrar & reflejar & abrir y cerrar \\
\hline
\end{tabular}

Nota. La letra normal indica la forma de los artefactos y la cursiva, la función que cumplen. 


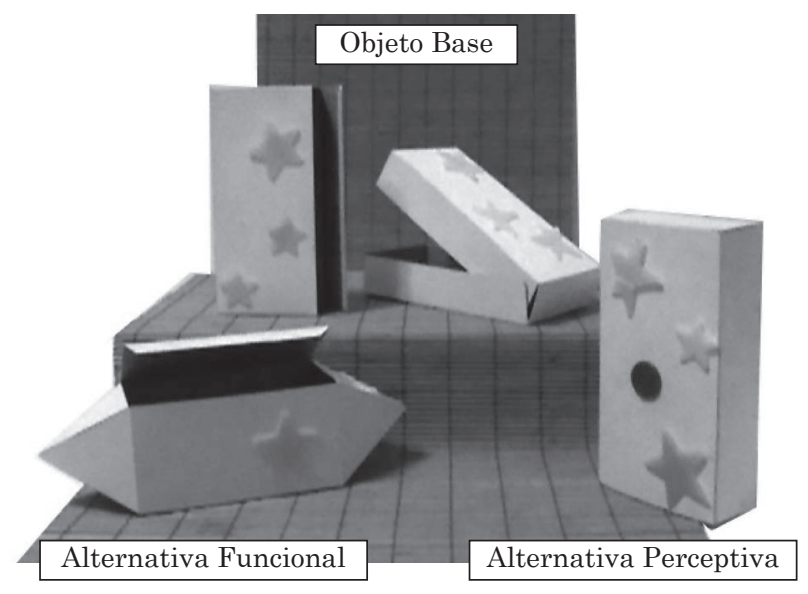

Figura 3. Estímulos utilizados en la condiciones con comparación.

Procedimiento. En la fase de ensayo presentamos los materiales (Marvin y su planeta). En las condiciones Con etiqueta dijimos a los niños que iban a aprender los nombres especiales de los objetos de Marvin. En las condiciones Sin etiqueta les dijimos que iban a jugar un juego con Marvin.

Después siguió la fase de evaluación. En la condición Comparación con etiqueta la experimentadora presentaba en cada subprueba dos artefactos modelo con una etiqueta artificial común y, señalando los ejemplares, invitaba al niño a establecer comparaciones, diciendo: "Mira, este es un Pong y este también es un Pong, mira lo que hacen los objetos que se llaman Pong", mostrando la función de los artefactos (e.g., abrir y cerrar). El objetivo era promover la detección de las similitudes funcionales entre los artefactos. Luego, la experimentadora pedía al niño que repitiera la palabra nueva. A continuación, presentaba los dos alternativas, mostrando sus respectivas funciones y, colocándolas sobre la mesa, decía: "Muéstrale a Marvin cuál de estos dos es también un Pong". Después de que el niño seleccionaba una alternativa, la experimentadora presentaba la subprueba siguiente.

En la condición Comparación sin etiqueta el procedimiento fue idéntico; solo cambiaba la manera de hacer referencia a los artefactos. La experimentadora presentaba al niño los dos artefactos modelo diciéndole: “iMira estos objetos que tiene Marvin! Mira lo que hace este [mostrando su función, e.g., abrir y cerrar] y mira lo que hace este [mostrando el otro artefacto modelo con su función, e.g. abrir y cerrar]. ¿Ves de qué manera los dos son del mismo tipo? [mostrando alternativamente la función de ambos]". Aquí también el objetivo era promover la detección de las similitudes funcionales. A continuación, la experimentadora presentaba las dos alternativas, mostrando sus respectivas funciones y, colocándolas sobre la mesa, preguntaba: “¿Puedes decirle a Marvin cuál de los dos es del mismo tipo que estos [artefactos modelo]?” Luego de que el niño seleccionaba una alternativa, la experimentadora presentaba la siguiente subprueba.

El procedimiento en la condición Sin comparación con etiqueta fue idéntico al del Estudio 1. El procedimiento en la condición Sin comparación sin etiqueta fue similar al del Estudio 1, solo que la experimentadora se refería al artefacto modelo sin nombrarlo: "iMira esto que tiene Marvin, mira lo que hace!", mostrando su función (e.g., abrir y cerrar). A continuación, presentaba las dos alternativas, mostrando sus respectivas funciones y, colocándolas sobre la mesa, preguntaba: “¿Puedes decirle a Marvin cuál de los dos [señalando las alternativas] es del mismo tipo que este [señalando el artefacto modelo]?” Una vez que el niño seleccionaba una alternativa, la experimentadora presentaba la siguiente subprueba. 
En ninguna de las condiciones los niños recibían corrección o feedback. El orden de presentación de las etiquetas y las subpruebas fue contrabalanceado. La ubicación derecha-izquierda de las alternativas (funcional-perceptiva) con relación al individuo fue asignada al azar en cada una de las subpruebas.

Análisis de Datos. Primero analizamos la proporción de respuestas funcionales (primera elección) contra el azar en cada condición, utilizando la prueba Binomial. Luego, valiéndonos de la prueba Kruskal-Wallis, comparamos la proporción de respuestas funcionales entre las condiciones. Posteriormente evaluamos las diferencias entre las condiciones por pares mediante la prueba U-Mann Whitney.

\section{Resultados}

En la condición Comparación con etiqueta los niños seleccionaron más alternativas funcionales que lo esperado por azar $(68,1 \%), p=0,003$. En el resto de las condiciones la ejecución de los niños fue al azar: Comparación sin etiqueta $(58,3 \%), p=0,19$, Sin comparación con etiqueta $(40,0 \%), p=0,12$, Sin comparación sin etiqueta $(38,9 \%), p=0,07$ (Tabla 3).

Tabla 3

Porcentaje de Respuestas Funcionales por Subprueba y Condición

\begin{tabular}{lcccc}
\hline \multirow{2}{*}{$\begin{array}{c}\text { Subprueba } \\
\text { (función) }\end{array}$} & \multicolumn{2}{c}{ Etiqueta } & \multicolumn{2}{c}{ Sin etiqueta } \\
\cline { 2 - 5 } & Comparación & Sin comparación & Comparación & Sin comparación \\
\hline Imantar & 50,0 & 33,3 & 58,0 & 33,3 \\
Verter & 83,3 & 25,0 & 50,0 & 41,7 \\
Guardar & 41,7 & 50,0 & 50,0 & 29,2 \\
Hacer ruido & 1,7 & 58,3 & 58,3 & 41,7 \\
Reflejar & 75,0 & 58,3 & 75,0 & 33,3 \\
Abrir y cerrar & 66,7 & 16,7 & 58,3 & 25,0 \\
Total & 68,1 & 40,3 & 58,3 & 38,9 \\
\hline
\end{tabular}

Encontramos diferencias significativas en la mediana de respuesta funcionales entre condiciones, $H(3, N=48) 17,3, p=0,001$. Análisis subsiguientes mostraron que en la condición Comparación con etiqueta los niños seleccionaron más respuestas funcionales que en las dos condiciones Sin comparación, tanto con etiqueta como en su ausencia: Comparación con etiqueta versus Sin comparación con etiqueta $U(1, N=24)=1872, p=0,001$; Comparación con etiqueta versus Sin comparación sin etiqueta $U(1, N=24)=1836, p=0,001$. Estos resultados revelan dos cuestiones. Primero, cuando los niños vieron solo un artefacto categorizaron azarosamente, aun cuando el artefacto recibió un nombre. Segundo, el confrontar a los niños con dos artefactos miembros de la misma categoría bajo un nombre común favoreció la extensión del nombre a alternativas funcionales.

En las condiciones Con comparación (con etiqueta-sin etiqueta), la proporción de respuestas funcionales varió significativamente, $U(1, N=24)=2340,2, p=0,02$. Asimismo, mientras que en la condición Con comparación sin etiqueta la ejecución de los niños fue al azar, en la condición Con comparación con etiqueta su ejecución superó el azar, lo cual sugiere que el nombre favoreció la detección de relaciones funcionales. 
Al comparar las condiciones Sin etiqueta (con y sin comparación), los niños seleccionaron más alternativas funcionales en la condición Con comparación que en la condición Sin comparación, $U(1, N=24)=2088, p=0,05)$. Esto sugiere que la oportunidad de comparar dos artefactos modelos en ausencia de un nombre común conduce a una atenuación del sesgo perceptivo, aunque no resultó ser suficiente para la detección de relaciones funcionales como lo fue con la presencia de un nombre.

\section{Discusión}

El estudio revela que la comparación de dos ejemplares categoriales que comparten un nombre permite ir más allá de la información perceptiva y categorizar objetos en base a relaciones más profundas, aun en ausencia de conocimiento y experiencia previa. Estos resultados extienden el rol de la comparación encontrado en estudios con categorías familiares en los que la yuxtaposición de objetos perceptiva y conceptualmente similares permite a los niños derivar la estructura conceptual que subyace a sus regularidades perceptivas (Gentner \& Namy, 1999; Namy \& Gentner, 2002).

Los resultados que presentamos muestran que el efecto de la comparación es tan poderoso que puede impulsar la utilización de la estructura conceptual subyacente también con objetos desconocidos, promoviendo la formación de categorías enteramente nuevas. Estos resultados se hallan en consonancia con evidencias que muestran que la especificidad del mecanismo de comparación es la alineación estructural (Namy \& Gentner, 2002); en el caso de este estudio, la función compartida por los artefactos miembros. Asimismo, apoyan la idea de que el efecto de la comparación en la categorización de artefactos no familiares parece ser específico de contextos lingüísticos, dado que, de todos los niños que participaron en las condiciones con comparación, solo los que recibieron la etiqueta fueron exitosos. Estos hallazgos son consistentes con los reportados por Graham et al. (2010) en cuanto al rol de la comparación y del lenguaje en la categorización de objetos no familiares en niños pequeños.

La comparación es una ruta que opera desde lo perceptual hacia la estructura conceptual subyacente. En el próximo estudio examinamos el rol de la inferencia en base a propiedades, proceso que pone en juego una ruta distinta que parte de lo conceptual, no ya de lo perceptivo, para poner en relieve el tipo conceptual de la categoría.

\section{Estudio 3}

En este estudio investigamos si los niños pequeños pueden inferir categorías nuevas de artefactos desconocidos a partir de sus propiedades específicas, como la función. Teniendo en cuenta que el Estudio 1 mostró que recién hacia los cinco años los niños utilizan por sí solos información funcional, decidimos evaluar el impacto de la inferencia en base a propiedades conceptuales en niños de cuatro años. Estudios realizados con categorías familiares han demostrado que la inferencia promueve la detección de relaciones supra-ordenadas en niños de esta edad (Gelman \& Markman, 1986).

Utilizamos la tarea de palabra extendida empleada en los estudios previos. Manipulamos experimentalmente la posibilidad de que los niños infirieran el tipo conceptual en base a la función de un artefacto desconocido, presentándoles ya sea el artefacto modelo con su respectiva función (condición con propiedades) o un artefacto modelo solo (condición sin propiedades), antes de extender la palabra a una alternativa. Nuestra hipótesis fue que si la inferencia en base a propiedades funcionales promueve la detección del tipo conceptual, los niños que reciben información sobre propiedades funcionales son más propensos a extender su nombre sobre la base de la función, mientras que los que no reciben esta información se focalizan predominantemente en relaciones perceptivas. 


\section{Método}

Participantes. En este estudio participaron 24 niños de cuatro años de edad en dos condiciones de 12 niños cada una: Con propiedades (47-52 meses, $M=49,0, D E=2,2)$ y Sin propiedades. Los datos de la condición Sin propiedades (47-49 meses, $M=48,3, D E=0,7$ ) provienen del grupo de niños de cuatro años del Estudio 1.

Materiales. Utilizamos los artefactos empleados en el Estudio 1. Una lista completa de los ítems de cada subprueba puede observarse en la Tabla 1.

Procedimiento. Los participantes fueron testeados en dos condiciones; ambas utilizaron la tarea de extensión de la palabra. Sin propiedades: procedimiento del Estudio 1. Con propiedades: además de la etiqueta, la experimentadora brindaba información explícita acerca de las propiedades funcionales de cada categoría, acompañada por una demostración. Por ejemplo: "Este es un Pong, los Pongs como estos sirven para abrir y cerrar ¿ves lo que pueden hacer los Pongs?”, mostrándole la función.

En ninguna condición los niños recibieron corrección o feedback. El orden de presentación de las series fue contrabalanceado. La ubicación derecha-izquierda de los estímulos alternativos y el orden de presentación de los objetos modelo fueron asignados al azar en cada una de las subpruebas.

Análisis de Datos. Mediante la prueba Binomial analizamos las respuestas contra el nivel del azar. Luego, utilizando la prueba U-Mann Whitney, examinamos la diferencia de ejecución entre las condiciones.

\section{Resultados}

Los niños seleccionaron respuestas funcionales al nivel del azar en ambas condiciones: con propiedades $(40,2 \%), p=0,10$, sin propiedades $(40,3 \%), p=0,10$ (Tabla 4). Las ejecuciones entre las condiciones no variaron, $U(1, N=24)=2592, p=0,100$.

Tabla 4

Porcentaje de las Alternativas Funcionales, por Condición y Subprueba

\begin{tabular}{lcc}
\hline \multirow{2}{*}{$\begin{array}{c}\text { Subprueba } \\
\text { (función) }\end{array}$} & \multicolumn{2}{c}{ Condición } \\
\cline { 2 - 3 } & Con propiedades & Sin propiedades \\
\hline Imantar & 25,0 & 33,3 \\
Verter & 25,0 & 25,0 \\
Guardar & 33,3 & 50,0 \\
Hacer ruido & 50,0 & 58,3 \\
Reflejar & 58,3 & 58,3 \\
Abrir y cerrar & 50,0 & 16,7 \\
Total & 40,2 & 40,3 \\
\hline
\end{tabular}




\section{Discusión}

Los resultados muestran que, aun cuando los niños recibieron información explícita (verbal y demostración) sobre las propiedades funcionales de los artefactos, no infirieron el tipo conceptual. Estos resultados concuerdan con los reportados por Farrar et al. (1992), quienes mostraron un bajo rendimiento en niños pequeños en procesos de inferencia relativos a conceptos de objetos desconocidos. Sin embargo, contradicen los hallazgos reportados por Truxaw et al. (2006), quienes encontraron una reducción de las respuestas perceptivas en relación a la información acerca de las propiedades funcionales de los artefactos. Una posible explicación de esta contradicción podría deberse a que en el estudio mencionado, además de las propiedades funcionales, los niños tuvieron la posibilidad de comparar los artefactos modelos con sus alternativas, estableciendo relaciones de similitud y contraste entre los mismos. Teniendo en cuenta los resultados del Estudio 2 que ilustran el efecto de la comparación como proceso analógico en la detección de la información funcional, es posible hipotetizar que en el trabajo de Truxaw et al. (2006) fue la comparación más que la inferencia en base a propiedades lo que contribuyó a la reducción de las respuesta perceptivas.

Si bien la inferencia en base a las propiedades funcionales ha demostrado ser una vía de conocimiento valiosa al extender aspectos del conocimiento conceptual que se posee (Gelman et al., 1986), este proceso no parece ser suficiente para promover un nuevo tipo conceptual con objetos no familiares. En este sentido, pareciera ser que la preexistencia de conocimiento sobre los objetos es un factor necesario para inferir un nuevo tipo conceptual a partir de propiedades, al menos en edades tempranas.

\section{Discusión General}

En la presente investigación indagamos la comparación y la inferencia en base a propiedades en la formación de categorías enteramente nuevas por parte de niños pequeños.

Hacia los cinco años los niños formaron categorías no familiares, categorizando artefactos desconocidos sobre la base de un conocimiento funcional y no solo de aspectos perceptivos. Antes de esa edad la mayoría de los niños dependió de aspectos perceptivos como base para extender el significado de un nombre. Sin embargo, utilizando el mecanismo de comparación, los niños de cuatro años pudieron sortear el sesgo perceptivo y formar categorías nuevas. Este mecanismo mostró ser eficiente para promover la información funcional que determina la estructura conceptual que subyace a las categorías de artefactos.

Por el contrario, la inferencia en base a propiedades no mostró ser una ruta eficiente para que los niños categorizaran artefactos desconocidos en base a su función. Al respecto, los estudios sobre conceptos familiares argumentan que el proceso de inferencia en base a propiedades opera a partir de propiedades conceptuales aplicadas a un ejemplar del concepto. Estas propiedades, apuntaladas por el repertorio de conceptos disponible en el equipamiento cognitivo de los niños, promueven las inferencias hacia un nuevo tipo conceptual (Gelman \& Coley, 1990). De ello se derivaría que, en ausencia de un conocimiento previo en el que apoyarse, la información brindada a los niños sobre las propiedades de los artefactos resultó ser insuficiente para despertar las inferencias necesarias para formar una categoría enteramente nueva.

Invocando trabajos previos acerca del rol de la comparación en el desarrollo de categorías (Namy \& Gentner, 2002), los resultados de la presente investigación revelan que este mecanismo posee una doble función: por un lado, sirve para enfatizar aspectos del conocimiento conceptual que se posee y, por otro, permite generar un conocimiento nuevo.

¿Cómo entender el alcance de la comparación en su doble función? La respuesta podría encontrarse en la ruta que este proceso emprende para acceder a la estructura conceptual. El yuxtaponer ejemplares de un concepto perceptiva y conceptualmente similares bajo un nombre 
común prescinde, desde su inicio, de la familiaridad con la categoría y su dominio conceptual específico. Solo en un segundo momento esa yuxtaposición perceptiva serviría de puente hacia una comprensión más profunda en cualquier dominio conceptual, sea familiar o no. Su independencia del dominio conceptual, al menos en un primer momento, abonaría la idea de que la comparación constituiría un proceso de aprendizaje de dominio general. Este supuesto se ve apoyado por numerosos estudios que han mostrado el impacto de la comparación en un amplio rango de aspectos del desarrollo cognitivo, incluyendo reconocimiento de rostros (Fagan III, 1978), tareas de conservación (Gelman, 1969), relaciones espaciales (Kotovsky \& Gentner, 1996) y aprendizaje de palabras (Waxman \& Klibanoff, 2000).

El caso de la inferencia, al partir de propiedades conceptuales, de entrada ubica al niño en la especificidad de la categoría; en el caso de los artefactos estudiados, en su función. Cuando el conocimiento disponible es fragmentario, como en el caso de las categorías no familiares, la información brindada no parece ser suficiente para sortear las propiedades perceptivas funcionalmente irrelevantes y extraer la estructura conceptual profunda.

En el marco de la comparación, la superioridad de la similitud perceptiva como puerta de entrada hacia una estructura conceptual nueva no implica necesariamente que los niños sean a-teóricos o que carezcan de interés por las propiedades funcionales. Numerosas investigaciones muestran que tempranamente los niños reconocen aspectos no obvios y profundos de los objetos (Markman \& Hutchinson, 1984; Waxman \& Gelman, 1986). Sin embargo, frente a la falta de conocimiento y experiencia previa, la ventaja de la comparación reside en su estrategia: hacer uso de una tendencia a enfatizar aspectos perceptivos, ya que los mismos pueden ser vaticinadores altamente confiables de las categorías (Namy \& Gentner, 2002).

En este trabajo hemos ilustrado la superioridad de la comparación solo en la promoción del conocimiento funcional de los objetos. Sin embargo, consideramos que este mecanismo podría ser eficiente en la promoción de otro tipo de conocimiento abstracto, además del funcional. Por ejemplo, en futuros trabajos sería importante evaluar el rol de la comparación en la promoción de conocimiento conceptual nuevo en base a relaciones causales entre objetos o eventos. Por otra parte, nuestros resultados mostraron que los niños de cuatro años sortearon el sesgo perceptivo, formando nuevas categorías, como resultado del proceso comparativo en un contexto lingüístico. Nos preguntamos si niños aun más pequeños podrían ser exitosos en la formación de categorías nuevas si son invitados a comparar artefactos miembros pero en un contexto de instrucción explícita. Estudios propios previos con categorías familiares mostraron que a los tres años los niños pudieron sortear el sesgo perceptivo y formar categorías supraordenadas, como resultado de la comparación y la inferencia, pero solo en un contexto socio-pragmático en el que recibían instrucción y feedback; no así cuando fueron invitados a comparar e inferir sin este soporte social (Taverna, 2009; Taverna \& Peralta, en prensa).

Para concluir, el desarrollo cognitivo se caracteriza por un inter-juego entre la experiencia y el conocimiento disponible en el repertorio cognitivo, por un lado, y los mecanismos o procesos de aprendizaje, por el otro. La formación de categorías es un ejemplo de ese inter-juego. Esta investigación muestra que, en ausencia de experiencia previa y con un conocimiento conceptual fragmentario o incompleto, la comparación, exponente del aprendizaje por analogía, es una ruta clave hacia la estructura conceptual profunda. 


\section{Referencias}

Baldwin, D. A. (1992). Clarifying the role of shape in children's taxonomic assumption. Journal of Experimental Child Psychology, 54, 392-416. doi:10.1016/0022-0965(92)90027-4

Booth, A. E., Waxman, S. R. \& Huang, Y. T. (2005). Conceptual information permeates word learning in infancy. Developmental Psychology, 41, 491-505. doi:10.1037/0012-1649.41.3.491

Bruner, J. S., Goodnow, J. J. \& Austin, C. A. (1956). A study of thinking. New York, NY: John Wiley \& Sons.

Diesendruck, G., Markson, L. \& Bloom, P. (2003). Children's reliance on creator's intent in extending names for artifacts. Psychological Science, 14, 164-168. doi:10.1111/1467-9280.t01-1-01436

Fagan III, J. F. (1978). Facilitation of infants' recognition memory. Child Development, 49, 1066-1075.

Farrar, M. J., Raney, G. E. \& Boyer, M. E. (1992). Knowledge, concepts, and inferences in childhood. Child Development, 63, 673-691. doi:10.1111/j.1467-8624.1992.tb01654.x

Gelman, R. (1969). Conservation acquisition: A problem of learning to attend to relevant attributes. Journal of Experimental Child Psychology, 7, 167-187. doi:10.1016/0022-0965(69)90041-1

Gelman, S. A. \& Coley, J. D. (1990). The importance of knowing a dodo is a bird: Categories and inferences in two-yearold children. Developmental Psychology, 26, 796-804. doi:10.1037/0012-1649.26.5.796

Gelman, S. A., Collman, P. \& Maccoby, E. E. (1986). Inferring properties from categories versus inferring categories from properties: The case of gender. Child Development, 57, 396-404.

Gelman, S. A. \& Markman, E. M. (1986). Categories and induction in young children. Cognition, 23, $183-209$. doi:10.1016/0010-0277(86)90034-X

Gentner, D. (1978). What looks like a jiggy but acts like a zimbo? A study of early word meaning using artificial objects. Papers and Reports on Child Language Development, 15, 1-6.

Gentner, D. \& Namy, L. L. (1999). Comparison in the development of categories. Cognitive Development, 14, 487-513. doi:10.1016/S0885-2014(99)00016-7

Gentner, D., Rattermann, M. J., Markman, A. \& Kotovsky, L. (1995). Two forces in the development of relational similarity. En T. J. Simon \& G. S. Halford (Eds.), Developing cognitive competence: New approaches to process modeling (pp. 263-313). Hillsdale, NJ: Lawrence Erlbaum.

Graham, S. A. \& Diesendruck, G. (2010). Fifteen-month-old infants attend to shape over other perceptual properties in an induction task. Cognitive Development, 25, 111-123. doi:10.1016/j.cogdev.2009.06.002

Graham, S. A., Kilbreath, C. S. \& Welder, A. N. (2004). Thirteen-month-olds rely on shared labels and shape similarity for inductive inferences. Child Development, 75, 409-427. doi:10.1111/j.1467-8624.2004.00683.x

Graham, S. A., Namy, L. L., Gentner, D. \& Meagher, K. (2010). The role of comparison in preschooler's novel object categorization. Journal of Experimental Child Psychology, 107, 280-290. doi:10.1016/j.jecp.2010.04.017

Hammer, R., Diesendruck, G., Weinshall, D. \& Hochstein, S. (2009). The development of category learning strategies: What makes the difference? Cognition, 112, 105-119. doi:10.1016/j.cognition.2009.03.012

Imai, M., Gentner, D. \& Uchida, N. (1994). Children's theories of word meaning: The role of shape similarity in early acquisition. Cognitive Development, 9, 45-75. doi:10.2307/1131354

Keil, F. C. (1996). Concepts, kinds, and cognitive development ( $3^{\mathrm{a}}$ ed.). Cambridge, MA: MIT Press.

Kemler Nelson, D. G., Herron, L. \& Morris, C. (2002). How children and adults name broken objects: Inferences and reasoning about design intentions in the categorization of artifacts. Journal of Cognition and Development, 3, 301-332. doi:10.1207/S15327647JCD0303_3

Kemler Nelson, D. G., Russell, R., Duke, N. \& Jones, K. (2000). Two-year-olds will name artifacts by their functions. Child Development, 71, 1271-1288. doi:10.1111/1467-8624.00228

Kotovsky, L. \& Gentner, D. (1996). Comparison and categorization in the development of relational similarity. Child Development, 67, 2797-2822. doi:10.1111/j.1467-8624.1996.tb01889.x

Loewenstein, J. \& Gentner, D. (2001). Spatial mapping in preschoolers: Close comparisons facilitate far mappings. Journal of Cognition and Development, 2, 189-219. doi:10.1207/S15327647JCD0202_4

Mandler, J. M. (1992). How to build a baby: II. Conceptual primitives. Psychological Review, 99, 587-604. doi:10.1037/0033-295X.99.4.587

Mandler, J. M. (2004). The foundations of mind: Origins of conceptual thought. New York, NY: Oxford University Press.

Mareschal, D. \& Quinn, P. C. (2001). Categorization in infancy. Trends in Cognitive Science, 5, 443-450. doi:10.1016/ S1364-6613(00)01752-6

Markman E. M. \& Hutchinson, J. E. (1984). Children's sensitivity to constraints on word meaning: Taxonomic versus thematic relations. Cognitive Psychology, 16, 1-27. doi:10.1016/0010-0285(84)90002-1

Namy, L. L. \& Gentner, D. (2002). Making a silk purse out of two sow's ears: Young children's use of comparison in category learning. Journal of Experimental Psychology: General, 131, 5-15. doi:10.1037//0096-3445.131.1.5

Sloutsky, V. M. (2003). The role of similarity in the development of categorization. Trends in Cognitive Science, 7, $246-$ 251. doi:10.1037/0096-3445.134.4.606

Taverna A. (2009). La mente paradigmática: desarrollo conceptual en contextos de colaboración (Tesis de Doctorado no publicada), Facultad de Psicología, Universidad Nacional de Córdoba, Argentina.

Taverna, A. \& Peralta, O. (2007). Desarrollo conceptual temprano: el impacto instruccional en la categorización de objetos. En M. C. Richaud \& M. S. Ison (Comps.), Avances en investigación en ciencias del comportamiento en Argentina (pp. 69-94). Mendoza, Argentina: Universidad del Aconcagua.

Taverna A. \& Peralta, O. (en prensa). Formación de categorías supraordenadas en la niñez temprana: el rol de la instrucción. Infancia y Aprendizaje. 
Truxaw, D., Krasnow, M. N., Woods, C. \& German, T. P. (2006). Conditions under which function information attenuates name extension via shape. Psychological Science, 17, 367-371. doi:10.1111/j.1467-9280.2006.01713.x

Vygotsky, L. S. (1962). Thought and language. Cambridge, MA: MIT Press.

Wang, S. \& Baillargeon, R. (2008). Can infants be "taught" to attend to a new physical variable in an event category? The case of height in covering events. Cognitive Psychology, 56, 284-326. doi:10.1016/j.cogpsych.2007.06.003

Waxman, S. \& Gelman, R. (1986). Preschoolers' use of superordinate relations in classification and language. Cognitive Development, 1, 139-156. doi:10.1016/j.cogpsych.2007.06.003

Waxman, S. R. \& Klibanoff, R. S. (2000). The role of comparison in the extension of novel adjectives. Developmental Psychology, 36, 571-581. doi:10.1037//0012-1649.36.5.571

Fecha de recepción: Junio de 2011.

Fecha de aceptación: Marzo de 2012. 